\title{
Síndrome de Wolff-Parkinson-White: uma revisão narrativa
}

\author{
Wolff-Parkinson-White Syndrome: a narrative review
}

Síndrome de Wolff-Parkinson-White: una revisión narrativa

Paula Ananda Chacon Inês ${ }^{1 *}$, Edson Alves Margarido', Ana Carolina Valadão Oliveira².

\section{RESUMO}

Objetivo: Os autores realizaram uma revisão bibliográfica a fim de conhecer os mais recentes aspectos genéticos, clínicos, diagnósticos e de tratamento dos portadores da síndrome de Wolff-Parkinson-White. Revisão bibliográfica: A síndrome de Wolff-Parkinson-White é uma patologia cardíaca na qual o paciente possui uma via de condução acessória e é diagnosticada unicamente por meio do eletrocardiograma. Embora alguns pacientes possam desenvolver sintomas, a maioria destes sãos assintomáticos e podem ter morte súbita como primeira manifestação da doença. O tratamento dos pacientes assintomáticos ainda é um assunto muito debatido no meio científico, devido às diretrizes não serem claras quanto a esses pacientes, associado à apresentação de estudos com resultados contraditórios. Considerações finais: $O$ melhor entendimento da apresentação clínica, do diagnóstico e dos tratamentos disponíveis para os portadores da síndrome de Wolff-Parkinson-White permitem reduzir a morbidade e a mortalidade dos pacientes, além de os tornarem ativos na decisão terapêutica.

Palavras-chave: Síndrome de Wolff-Parkinson-white, Ablação por cateter, Arritmia.

\begin{abstract}
Objective: The authors performed a bibliographic review in order to know the latest genetic, clinical, diagnostic and treatment aspects of patients with Wolff-Parkinson-White syndrome. Bibliography review: WolffParkinson-White syndrome is a cardiac pathology in which the patient has an accessory conduction pathway and is diagnosed solely by electrocardiogram. Although some patients may develop symptoms, most of them are asymptomatic and may have sudden death as the first manifestation of the disease. The treatment of asymptomatic patients is still a hotly debated issue in the scientific world because the guidelines are not clear about these, associated with the presentation of studies with contradictory results. Final Considerations: A better understanding of the clinical presentation, diagnosis and treatments available to patients with WolffParkinson-White syndrome allows the improvement of patients' morbidity and mortality, as well as making them active in the therapeutic decision.
\end{abstract}

Keywords: Wolff-Parkinson-White syndrome, Catheter ablation, Arrhythmia.

\section{RESUMEN}

Objetivo: Los autores realizaron una revisión bibliográfica para conocer los últimos aspectos genéticos, clínicos, diagnósticos y de tratamiento de pacientes con síndrome de Wolff-Parkinson-White. Revisión bibliográfica: El síndrome de Wolff-Parkinson-White es una patología cardíaca en la que el paciente tiene una vía de conducción accesoria y se diagnostica únicamente mediante un electrocardiograma. Aunque algunos pacientes pueden desarrollar síntomas, la mayoría de ellos son asintomáticos y pueden tener muerte

${ }_{1}^{1}$ Centro Universitário Municipal de Franca (Uni-FACEF), Franca - SP

2 Universidade de Franca (UNIFRAN), Franca - SP

*E-mail: paulachaconn@gmail.com. 
súbita como la primera manifestación de la enfermedad. El tratamiento de pacientes asintomáticos sigue siendo un tema muy debatido en el mundo científico porque las pautas no son claras al respecto, asociadas con la presentación de estudios con resultados contradictorios. Consideraciones finales: Una mejor comprensión de la presentación clínica, el diagnóstico y los tratamientos disponibles para los pacientes con síndrome de Wolff-Parkinson-White permite mejorar la morbilidad y mortalidad de los pacientes, además de hacerlos activos en la decisión terapéutica.

Palabras clave: Síndrome de Wolff-Parkinson-White, Ablación con catéter, Arritmia.

\section{INTRODUÇÃO}

A síndrome conhecida como Wolff-Parkinson White (WPW) é uma condição cardíaca na qual o sistema de condução elétrico do coração apresenta uma ou mais vias acessórias e essas conduzem o estímulo elétrico mais rapidamente que o percorrido pelo nó atrioventricular, podendo ocasionar episódios de arritmias. Apesar de já terem sido realizadas diversas buscas em alterações genéticas que justificassem o aparecimento desta patologia, pouco se sabe sobre o assunto, principalmente quando a mesma não tem relação familiar (QIU M, et al., 2018).

Estudos e relatos de caso documentaram a presença de sintomas como taquicardia supraventricular, palpitações e síncope em alguns pacientes portadores da síndrome de Wolff-Parkinson-White, porém a maioria dos pacientes são assintomáticos e a dificuldade diagnóstica esbarra na existência do exame de eletrocardiograma como único meio para realizá-lo; contudo, as vias acessórias podem ser únicas ou múltiplas e visíveis ou não ao exame eletrocardiográfico, dificultando, desta forma, ainda mais seu achado (ETHERIDGE SP, et al., 2018). Diante disto, questiona-se o uso do mapeamento cardíaco detalhado por meio de estudo eletrofisiológico para que se estratifique fatores de risco e a real necessidade de medidas terapêuticas diante de portadores da patologia (SOO WM, et al., 2011).

A maioria dos pacientes portadores assintomáticos da síndrome de Wolff-Parkinson White apresentam um bom prognóstico a longo prazo, porém não se pode descartar o risco de uma pior evolução com arritmias ou até mesmo morte súbita, desta forma, muito se pergunta sobre qual a maneira certa de tratá-los, através de método invasivo como a ablação por cateter de radiofrequência ou método conservador, uma vez que podem ser assintomáticos pelo resto da vida, sendo incerto suas evoluções (SILVA G, et al., 2013; BELHASSEN B e GLIKSON M, 2010).

Devido ao fato de as diretrizes não serem claras quanto à conduta em pacientes assintomáticos, a maneira mais correta deve ser apresentar aos atingidos pela doença todas as alternativas disponíveis de tratamento, com seus riscos e benefícios, e considerar sua vontade no momento para estabelecer uma terapêutica conjunta (LIU A e PUSALKAR P, 2011). Embora a ablação por cateter possa reduzir a evolução maligna no futuro, deve-se sempre levar em conta o risco, mesmo que baixo, das complicações pela qual estão sujeitos os pacientes que se submetem a tal procedimento, além da possibilidade de não cura ou desenvolvimento de novas arritmias (ETHERIDGE SP, et al., 2018).

Desta maneira, o objetivo desta revisão bibliográfica é conhecer os mais recentes aspectos genéticos, clínicos, diagnósticos e, principalmente, de tratamento dos portadores da síndrome de Wolff-Parkinson-White, a fim de buscar uma redução na morbidade e na mortalidade dos mesmos.

\section{REVISÃO BIBLIOGRÁFICA}

\section{Descrição e definição da doença}

A síndrome de Wolff-Parkinson White (WPW) é descrita como uma patologia cardíaca na qual o sistema elétrico de condução apresenta uma ou mais vias acessórias, fazendo com que a corrente elétrica que promove as contrações do coração passe por essas de uma forma rápida e não pausada, ao contrário do que ocorreria no nó atrioventricular, predispondo o paciente ao desenvolvimento de arritmias (QIU M, et al., 2018). 
Muitos estudos foram feitos na busca por alterações genéticas que justificassem o desenvolvimento da doença e parte deles identificaram um gene nomeado como PRKAG2 que possivelmente pode estar relacionado à suscetibilidade em desenvolver a síndrome de Wolff-Parkinson-White. Porém, este gene estaria ligado apenas à patologia de caráter familiar; desta forma, não são todos os pacientes que apresentariam alterações na avaliação genômica, uma vez que parte destes apresentam a doença sem relação familiar, dificultando ainda mais o diagnóstico (QIU M, et al., 2018).

\section{Alteração genética e sua fisiopatologia}

Embora a grande maioria dos portadores da síndrome de Wolff-Parkinson White possuam a patologia adquirida, pequena parcela deles apresentam a síndrome com caráter familiar associada a hipertrofia cardíaca. Apesar dos mecanismos moleculares que levam à formação de uma via acessória ainda não estarem muito bem elucidados, sabe-se que se trata de uma forma autossômica dominante relacionada a mutações no gene PRKAG2, responsável pela codificação de uma subunidade da 5'AMP-proteina quinase ativada (AMPK). Atualmente, sabe-se que ao menos seis mutações do gene PRKAG2 estão associadas à síndrome de Wolff-Parkinson White familiar e o impacto de cada mutação ainda não foi bem esclarecido, tornando os fenótipos dependentes da maneira em que ocorre a formação de vias acessórias associadas a alterações na condução elétrica (MIYAMOTO L, 2018; STELD LP, et al., 2017).

A 5'AMP-proteina quinase ativada (AMPK) tem o papel de regular a homeostase energética, atuando como um sensor que tem seu papel desempenhado pela regulação da adenosina trifosfato (ATP) através da via de oxidação de ácidos graxos e captação de glicose. Acredita-se que a mutação do gene PRKAG2 leva ao desenvolvimento da síndrome de Wolff-Parkinson-White familiar pelo acúmulo de glicogênio em vacúolos intracelulares dos miócitos, o qual seria capaz de modificar a condução elétrica atrioventricular e induzir a pré-excitação, porém ainda não há evidências que demonstram o acúmulo de glicogênio entre os átrios e os ventrículos, deixando bastante controversa a relação entre AMPK e a síntese de glicogênio (BANANKHAH P, et al., 2018).

Desta forma, torna-se evidente a importância de maiores estudos relacionados ao acúmulo cardíaco de glicogênio e à regulação dos canais iônicos, além da sua relação com o desenvolvimento de Wolff-Parkinson White (MIYAMOTO L, 2018).

\section{Apresentação clínica}

Por meio de relatos de caso e estudos disponíveis na literatura atual, sabe-se que grande parte dos pacientes portadores da síndrome de Wolff-Parkinson White são assintomáticos e descobrem a patologia ocasionalmente em exames eletrocardiográficos solicitados por outras razões, porém uma parte dos portadores apresentam sintomas que se manifestam como palpitações, tonturas e até mesmo morte súbita (QIU M, et al., 2018). Casos de fibrilação atrial são muito comuns nestes pacientes e, caso o portador desta patologia tenha uma via acessória com um período refratário muito curto e apresente um episódio de fibrilação atrial, esta leva a uma rápida condução aos ventrículos de forma repetitiva, ocasionando fibrilação ventricular e consequentemente parada cardíaca, podendo esta ser a primeira manifestação da doença (SILVA G, et al., 2013).

STEC SM, et al. (2014), em seu estudo, trouxe como evidência que alguns relatos de caso já demonstraram um padrão de Wolff-Parkinson White associado ao desenvolvimento de cardiomiopatia e até mesmo associação entre pré-excitação e desempenho cardiopulmonar diminuído. Durante o estudo foram relatados diversos casos de pacientes que apresentavam como manifestação uma dispneia crônica inexplicável por outra patologia e associado a isso havia um padrão Wolff-Parkinson White no eletrocardiograma (ECG). O mecanismo da dispneia em pacientes com pré-excitação ainda precisa de maiores estudos, mas acredita-se que seja através de mecanismos de dissincronia ventricular e por receptores cardiopulmonares e, com isso, acredita-se que futuramente esse sintoma possa ser incluído juntamente com aqueles já utilizados para classificar um paciente portador as síndromes como sintomático.

A literatura mais recente demonstra, além dos sintomas já expostos, uma possível relação entre a presença da síndrome de Wolff-Parkinson White e o desenvolvimento de insuficiência cardíaca. Acredita-se 
que a pré-excitação ventricular que corre pela via acessória seja capaz de promover uma pré carga diminuída, devidos as contrações precoces, com isso diminuindo a tensão de trabalho do músculo cardíaco, predispondo ao afinamento da parede do coração, o que, após um tempo, levaria à dilatação do segmento durante períodos de contração e, consequentemente, ocasionaria desenvolvimento de insuficiência cardíaca (LEE HJ, et al., 2018).

A partir de um estudo realizado no ano de 2017, através da revisão de bancos de dados de pacientes portadores de Wolff-Parkinson White com morte súbita, foi possível observar que, apesar de grande parte deles serem portadores sintomáticos, alguns podem evoluir ao óbito de forma repentina mesmo na ausência de sintomas e, por meio de estudos, tem-se que o risco de morte súbita em pacientes portadores da síndrome de Wolff-Parkinson White é aproximadamente de $0,1 \%$ em pacientes assintomáticos e $0,3 \%$ em pacientes sintomáticos por ano (FINOCCHIARO G, et al., 2017; QIU M, et al., 2018).

Embora a morte súbita nesta patologia seja muitas vezes silenciosa ou, em alguns casos, associada a outras alterações cardíacas, já foi evidenciado que a mesma ocorre com maior frequência durante períodos de sono ou descanso, mostrando, desta forma, que a restrição esportiva não evitaria piores evoluções da doença.

Contudo, tendo-se como análise que o tempo gasto com atividades esportivas e o tempo gasto em repouso são de grande desproporcionalidade, não se pode assegurar que pacientes portadores de síndrome de WolffParkinson White pratiquem atividades físicas de forma irrestrita, recomendando que sejam evitados esportes competitivos, todavia também não se pode afirmar que a restrição total aos esportes manteria os pacientes seguros, devendo dessa forma ser orientado quando a avaliações periódicas destes pacientes para realização de atividade física (ETHERIDGE SP, et al., 2018).

\section{Diagnóstico}

Infelizmente, o único recurso existente para o diagnóstico de Wolff-Parkinson White ainda é o eletrocardiograma. Em um paciente sem a patologia, o tempo entre a despolarização dos átrios e o atraso no nó atrioventricular, representado pelo segmento PR no ECG, tem uma duração de 0,12 segundos até 0,20 segundos e a despolarização dos ventrículos que se segue, representado pelo QRS no ECG, tem uma duração de no máximo 0,12 segundos e uma elevação rápida.

A representação eletrocardiográfica de um paciente portador de Wolff-Parkinson White apresenta-se com redução no intervalo PR (menor de 0,12 segundos), alargamento do complexo QRS (com duração total maior de 0,12 segundos) e onda delta (representada graficamente como uma curva de elevação inicial lentificada no complexo QRS) (QIU M, et al., 2018) (Figura 1 e 2).

Figura 1 - Representação gráfica de um eletrocardiograma sem alterações.

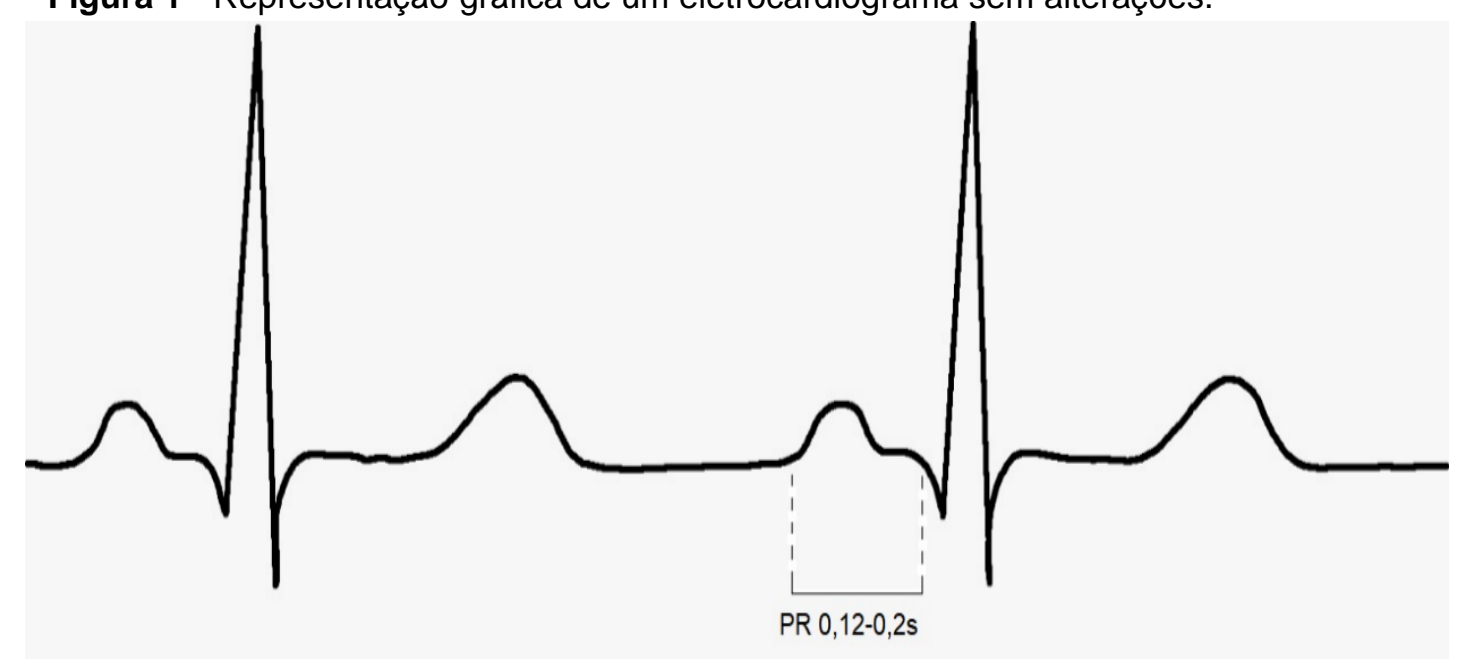

Fonte: Inês PAC, et al., 2020. 
Figura 2 - Representação gráfica de um eletrocardiograma de um paciente portador da síndrome de Wolff-Parkinson-White com identificação da onda delta e redução do intervalo PR.

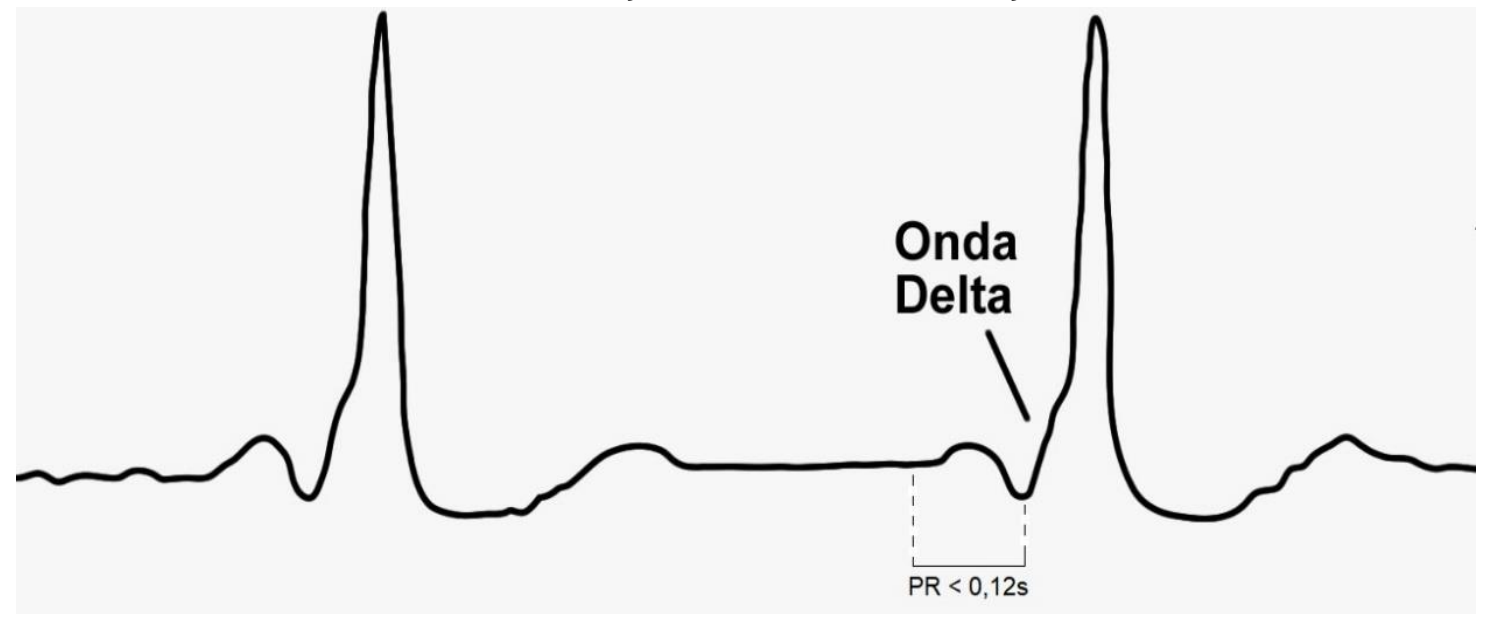

Fonte: Inês PAC, et al., 2020.

Todas essas alterações características da síndrome de Wolff-Parkinson-White são devidas à condução elétrica utilizar a via acessória para chegar aos ventrículos, a qual contorna o nó atrioventricular, fazendo com que o impulso atinja os ventrículos rapidamente, ocasionando uma despolarização precoce.

Além disso, em se tratando de fisiologia cardíaca, a frequência intrínseca no nó atrioventricular é de aproximadamente 180-200 batimentos por minuto e, diante de um paciente com frequência acima desta, devem-se suspeitar imediatamente da possibilidade de uma taquicardia ortodrômica reentrante com um componente de reentrada atrioventricular, como a síndrome de Wolff-Parkinson White (KESLER K e LAHHAM S, 2016).

Diferentemente de portadores sintomáticos que são mais evidentes os riscos e as apresentações, a avaliação de risco em pacientes assintomáticos ainda não é um consenso e, diante disso, alguns fatores de risco para evolução desfavorável do quadro deveriam ser avaliados em todos os pacientes. Embora o estudo eletrofisiológico possa auxiliar de forma confiável na avaliação de risco, o mesmo ainda não é amplamente disponível e utilizado, por se tratar de um método invasivo (ROSENHECK S, 2010).

Alguns marcadores não invasivos, como perda da condução na via acessória durante teste de estresse e após uso de drogas antiarrítmicas como procainamida e flecainida, foram colocadas em questão, mas rapidamente evidenciou-se que sua sensibilidade não era suficiente e tinham pouco papel na seleção de pacientes com alto risco. Quando se lança mão do estudo eletrofisiológico, identificou-se que período refratário efetivo da via acessória menor que 250 milissegundos, taquiarritimias durante o estudo eletrofisiológico, múltiplas vias acessórias, sexo masculino, idade, principalmente crianças, e presença de síncope estão diretamente relacionadas ao alto risco de evolução com pior prognóstico (SILVA G, et al., 2013; OZENC S, et al., 2014).

Além do estudo eletrofisiológico, estudos foram feitos com o uso do ecocardiograma tridimensional na tentativa de auxílio na avaliação de risco, uma vez que essa ferramenta fornece informações da deformação miocárdica e da força de contração do músculo cardíaco.

Infelizmente, foi identificado que esta técnica não consegue substituir a análise invasiva, a qual permanece sendo padrão-ouro, por mostrar apenas ativação mecânica e não elétrica, identificando de maneira aproximada a localização da via acessória, sendo a mesma correspondente na imagem como uma contração anormal do coração (ISHIZU T, et al., 2019; MARECHAUX S, 2019).

Um dos motivos que despertam interesse dos médicos no estudo dos pacientes assintomáticos com a síndrome de Wolff-Parkinson White, principalmente a partir do estudo eletrofisiológico, foi a possibilidade da ablação por cateter da via acessória destes, contribuindo na morbidade e mortalidade dos portadores de 
Wolff-Parkinson White, tornando uma possibilidade definitiva de tratamento. Contudo, diante desses pacientes, deve-se realizar alguns questionamentos para ver a real validade de tratamento na ausência de sintomas, como a existência dos fatores de risco já mencionados e sujeitos envolvidos em ocupações de alto risco (piloto, bombeiro, esportistas competitivos), além de avaliar o equilíbrio desta com as possíveis complicações associadas à ablação (ROSENHECK S, 2010; BELHASSEN B e GLIKSON M, 2010).

\section{Tratamento}

Já se tem conhecimento que a ablação por cateter de radiofrequência das vias acessórias é uma das opções e, na maioria das vezes, a de escolha para aqueles pacientes que possuem uma via acessória entre os átrios e os ventrículos, permitindo que sejam realizados bloqueios no trajeto elétrico, impedindo que a condução seja concluída (MUJOVIC N, et al., 2011). Este método de tratamento é bem estabelecido em pacientes sintomáticos com síndrome de Wolff-Parkinson White, uma vez que o objetivo é abolir os sintomas, porém a abordagem em pacientes assintomáticos, com o intuito de redução dos episódios de morte súbita, é menos conclusiva, tendo a ablação indicação classe lla de acordo com recomendações da American College of Cardiology / American Heart Association / European Society of Cardiology (ACC/AHA/ESC) (SOO WM, et al., 2011).

O procedimento de ablação por cateter de radiofrequência, apesar de ser um método invasivo, tem sua eficácia próxima a 100\% e uma mortalidade relacionada ao procedimento inferior a $0,2 \%$ quando realizada por profissional experiente (SILVA G, et al., 2013); porém, apesar de baixos riscos, não é isento destes, uma vez que os pacientes podem apresentar complicações, como perfuração, lesão valvar, tamponamento e eventos trombóticos, as quais são conhecidas por meio de relatos de casos apresentados nas bases de dados (LIU A e PUSALKAR P, 2011) (HIRATA K, et al., 2015). Além disso, pela necessidade de profissionais capacitados para minimizar os riscos do procedimento e seus custos, muitos locais ainda encontram entraves no estabelecimento do procedimento de maneira profilática em portadores assintomáticos (SILVA G, et al., 2013).

A literatura mais recente traz uma controvérsia em relação aos reais benefícios do tratamento por ablação nos portadores da síndrome de Wolff-Parkinson White, principalmente quando a mesma é realizada em portadores assintomáticos de maneira profilática. Alguns estudos demonstraram que o uso da terapia de ablação de maneira profilática está relacionado a uma diminuição do risco de arritmias malignas no futuro (SILVA G, et al., 2013).

Todavia, outros estudos de pacientes portadores assintomáticos da síndrome de Wolff-Parkinson White evidenciaram que a ablação por cateter de radiofrequência de maneira profilática não mostrou redução significativa em cinco anos na chance de evolução para arritmia fatal (OZENC S, et al., 2014), além da possibilidade de aparecimento de novas arritmias (MUJOVIC N, et al., 2011), tal fato podendo se justificar pela possibilidade da existência de múltiplas vias ou até mesmo de outros fatores somados e que contribuem para arritmias fatais, uma vez que parte substancial dos pacientes apresentam outra patologia cardíaca simultaneamente (FINOCCHIARO G, et al., 2017).

Além das discussões acerca do real benefício da ablação por cateter, alguns estudos demonstraram que grande parte dos pacientes portadores assintomáticos da síndrome de Wolff-Parkinson White apresentam sintomas como taquicardia e síncope, antes de apresentar um episódio de fibrilação ventricular e evolução para morte súbita, permitindo desta forma que a educação dos pacientes seja uma maneira eficaz de prevenção das evoluções malignas. Desta maneira, haveria um tempo de reconhecimento dos sintomas premonitórios, chegada ao serviço de emergência logo no início do quadro e tratamento em tempo hábil (OBEYESEKERE MN e KLEIN GJ, 2019). Diante destes achados, a decisão de eliminar a via acessória com o método de ablação deve ser discuto e o paciente deve ser informado sobre a eficácia do procedimento, a possibilidade de não cura e também sobre seus riscos de complicações (THE AMERICAN HEART ASSOCIATION AND THE HEART RHYTHM SOCIETY, 2016).

Diante do maior risco de evolução de pior prognóstico em crianças e da não possibilidade de queixa dos sintomas pela dificuldade de expressão dos mesmos, muito se estuda sobre a abordagem conservadora ou 
invasiva nesta faixa etária. Relatos de casos de pacientes pediátricos demonstraram que o uso com cautela da flecainida, um antiarrítmico classe Ic, pode tornar-se uma opção terapêutica nos pacientes com síndrome de Wolff-Parkinson White. Sabe-se que o uso desta medicação na população adulta acarreta maiores eventos arrítmicos e até aumento das hospitalizações em pacientes com fibrilação atrial, porém foi demonstrado ser eficaz na população pediátrica, embora ainda tenha que se ter cuidado naqueles com função cardiovascular comprometida, devido aos efeitos inotrópicos do medicamento (SEKINE M, et al., 2019).

Além disso, a dificuldade e a necessidade de avaliação cuidadosa na indicação do procedimento invasivo nessa população se dão pelo tamanho do paciente, o que dificulta a disponibilidade de cateteres adequados, tornando maior o risco de complicações (GONZALEZ MD, et al., 2018; ETHERIDGE SP, et al., 2018).

Associado a isso, há o fato de que um estudo avaliou pacientes pediátricos durante cinco anos após a realização da ablação e constatou que a eliminação da via acessória se deu em apenas $80,7 \%$ destes, com recorrência nos demais, seja precoce ou tardia, e aqueles pacientes que não foram submetidos a ablação permaneceram assintomáticos (GONZALEZ MD, et al., 2018). Desta forma, tem-se que a abordagem no paciente pediátrico deve ser cuidadosamente selecionada e avaliada em todas as opções disponíveis.

\section{CONSIDERAÇÕES FINAIS}

A síndrome de Wolff-Parkinson-White é um diagnóstico que deve ser lembrado quando feita a avaliação eletrocardiográfica de um paciente, mesmo que na ausência de sintomas, uma vez que a morte súbita pode ser a primeira manifestação. Diante deste achado, deve-se alertar os portadores sobre os possíveis sintomas que podem surgir e a necessidade da ida ao hospital rapidamente na vigência destes. Além disso, embora muito questionado ainda, deve-se apresentar ao paciente as opções disponíveis para tratamento, juntamente com os riscos e benefícios inerentes a esse, para que se tome uma conduta em conjunto na busca da melhora da morbidade e mortalidade dos portadores da doença. Com todos os achados, esta revisão mostra a necessidade de maiores estudos acerca do tema e da real validade de indicação de ablação por cateter de radiofrequência em portadores assintomáticos, uma vez que ainda são muito controversos seus benefícios.

\section{REFERÊNCIAS}

1. AL-KHATIB SM, et al. Risk Stratification for Arrhythmic Events in Patients With Asymptomatic Pre-Excitation: A Systematic Review for the 2015 ACC/AHA/HRS Guideline for the Management of Adult Patients With Supraventricular Tachycardia. Journal of the American College of Cardiology, 2016; 67(13): 1624-1638.

2. BANANKHAH P, et al. Cardiac manifestations of PRKAG2 mutation. BMC Medical Genetics, 2018; $19(1): 1-4$.

3. BELHASSEN B, GLIKSON M. Electrophysiological Testing and Ablation in an Asymptomatic Child with WolffParkinson-White Syndrome. The Israel Medical Association Journal, 2010; 12: 707-708.

4. ETHERIDGE SP, et al. Life-Threatening Event Risk in Children With Wolff-Parkinson-White Syndrome: A Multicenter International Study. JACCClinical Electrophysiology, 2018; 4: 433-444.

5. FINOCCHIARO G, et al. Sudden Cardiac Death in Pre-Excitation and Wolff-Parkinson-White: Demographic and Clinical Features. Journal of the American College of Cardiology, 2017; 69(12): 1644-1645.

6. GONZALEZ MD, et al. Catheter Ablation in Children With Accessory A-V Pathway: Do Short-Term Results Predict Long-Term Success?. Journal of the American College of Cardiology, 2018; 4: 456-458.

7. HIRATA K, et al. Left ventricular free wall perforation and left atrial dissection: unusual complication of radiofrequency catheter ablation for Wolf-Parkinson-White syndrome. British Medical Journal, 2015; 21: 1-4.

8. ISHIZU T, et al. Noninvasive Localization of Accessory Pathways in Wolff-Parkinson-White Syndrome by ThreeDimensional Speckle Tracking Echocardiography. Circulation: Cardiovascular Imaging, 2019; 1-9.

9. KESLER K e LAHHAM S. Tachyarrhythmia in Wolff-Parkinson-White Syndrome. The Western Journal of Emergency Medicine, 2016; 17(4): 469-470.

10. LEE HJ, et al. Altered myocardial characteristics of the preexcited segment in Wolff-Parkinson-White syndrome: A pilot study with cardiac magnetic resonance imaging. Plos One, 2018; 13(6): 1-13.

11. LIU A, PUSALKAR P. Asymptomatic Wolff-Parkinson-White syndrome: incidental ECG diagnosis and a review of literature regarding current treatment. British Medical Journal, 2011; 10: 1-4.

12. MARECHAUX S. The Wolff-Parkinson-White Syndrome: A Test Bed for the Assessment of Myocardial Dyssynchrony?. Circulation: Cardiovascular Imaging, 2019; 1-3. 
13. MIYAMOTO L. Molecular Pathogenesis of Familial Wolff-Parkinson-White Syndrome. Molecular Mechanisms of Cardiac Glycogen Regulation by AMPK. The Journal of Medical Investigation, 2018; 65: 1-8.

14. MUJOVIC N. et al. The Occurrence of New Arrhythmias after Catheter-Ablation of Accessory Pathway: Delayed Arrhythmic Side-Effect of Curative Radiofrequency Lesion?. Srpski Arhivza Celokupno Lekarstvo, 2011; 139(7): 458464.

15. ROSENHECK S. The Mystery of Asymptomatic Wolff-Parkinson-White Syndrome. The Israel Medical Association Journal, 2010; 12: 701-702.

16. SILVA G, et al. Aborted sudden cardiac death as first presentation of Wolff-Parkinson-White syndrome. Revista Portuguesa de Cardiologia, 2013; 32(4): 325-329.

17. SEKINE M, et al. Improvement in Dyssynchrony with Pharmacological Ablation of Right-Sided Accessory PathwayInduced Cardiomyopathy in Infants. International Heart Journal, 2019; 60: 1201-1205.

18. STEC SM, et al. Dyspnea related to pre-excitation during sinus rhythm as a new indication for catheter ablation. Kardiologia Polska, 2014; 72(7): 646-653.

19. STELD LP, et al. Wolff-Parkinson-White Syndrome with Ventricular Hypertrophy in a Brazilian Family. American Journal of Case Reports, 2017; 18: 766-776.

20. SOO WM, et al. ECG Delta waves in patients with palpitation. Singapore Med J, 2011; 52(2) 68-72.

21. OBEYESEKERE MN, KLEIN GJ. The Asymptomatic Wolff-Parkinson-White Patient: Time to Be More Proactive?. Circulation: Cardiovascular Imaging, 2019; 130: 805-807.

22. OZENC S, et al. Prophylactic accessory-pathway ablation in asymptomatic patients with a Wolff-Parkinson-White electrocardiographic pattern. European Review for Medical and Pharmacological Sciences, 2014; 18: 981-984. 